\title{
Influenza B outbreak in a primary school in Adelaide, Australia, 2011
}

\author{
Louise Flood, a Jane Raupach, ${ }^{a}$ Katina D’Onise ${ }^{a}$ and Deidre Russo ${ }^{a}$ \\ Correspondence to Louise Flood (e-mail: louise.flood@health.sa.gov.au)
}

Introduction: This report describes a 2011 seasonal influenza B outbreak in a metropolitan primary school in Australia with 179 students.

Methods: Epidemiological, microbiological and environmental investigations were undertaken. A retrospective cohort study was conducted using a questionnaire that included demographic data, details of illness, chronic health conditions and vaccination status. Influenza-like illness (ILI) was defined as fever plus cough and/or sore throat. Analysis of ILI was undertaken with the chi-squared test and Fisher's exact test.

Results: Seventy-two questionnaire respondents (75\%) reported illness during the outbreak - 43 with ILI, giving an attack rate of $45 \%$. There was no association between ILI and age or chronic lung disease. Six (6\%) students were vaccinated against influenza before the outbreak; although four became ill, none satisfied the ILI case definition. Seven students were positive for influenza B including two confirmed as B/Brisbane/60/2008-like; one student was positive for rhinovirus and another for metapneumovirus. The recommended influenza vaccine matched the circulating influenza strains.

Discussion: This cohort study estimated a high ILI attack rate and demonstrated low influenza vaccine coverage within the setting of a primary school. Gastrointestinal symptoms, in addition to constitutional and respiratory symptoms, were common.

S easonal influenza outbreaks can cause substantial health burden, through both morbidity and mortality in all age groups, that can overwhelm health services. ${ }^{1}$ Influenza in children who attend school or childcare is central to the community spread of influenza and epidemic amplification. ${ }^{2-4}$ Despite this, there is limited information in the peer-reviewed literature on the behaviour of seasonal influenza outbreaks in day schools. $^{5-7}$

Influenza is a notifiable disease in South Australia $(\mathrm{SA})$. Doctors and laboratories are required to report suspected or confirmed influenza to the Communicable Disease Control Branch (CDCB) of the South Australian Department for Health and Ageing; ${ }^{8,9}$ however, this is likely to under-represent the true extent of influenza infection in the community.

In SA, seasonal influenza usually peaks over the winter and spring (June to November) months. ${ }^{10}$ An average of 61 cases per month were notified from January to April 2011. In 2011, 4790 influenza cases were notified. ${ }^{11}$

On Thursday, 26 May 2011, CDCB was notified by the principal of an Adelaide metropolitan primary school (students aged five to 10 years) of a large number of unwell students (106 out of 179 students since 23 May 2011) and one unwell staff member. The illness was reported as one-to-two day gastroenteritislike illness with headache, vomiting and fever (but no diarrhoea), respiratory symptoms in some students, as well as reports of transmission within families. There had been no recent excursions or camps, no combined assemblies and the school did not have a canteen. On the advice of $C D C B$, the school sent home information regarding the outbreak and placed notices with advice to stay home if unwell.

An investigation was initiated by $C D C B$ to further characterize the illness by symptomatology and etiology and to estimate the attack rate and vaccine effectiveness within this school cohort.

\section{METHODS}

\section{Epidemiological investigation}

Due to confidentiality issues, a list of children who attended the school was unable to be provided to CDCB. Initially, the school contacted parents of ill children and requested they contact CDCB. From this, preliminary

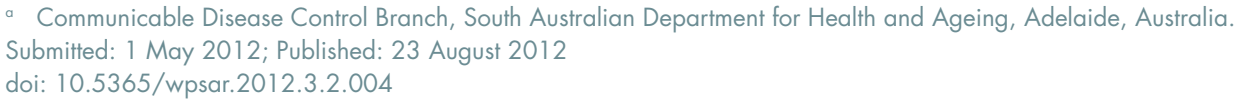


telephone interviews were conducted with parents of 10 ill children to determine demographic details, symptoms, management and details of ill contacts. Active case finding was also conducted by telephoning local medical practitioners. Doctors who notified local children with laboratory-diagnosed influenza were contacted to obtain clinical history. Parents of locally residing children aged five to 10 years with notified influenza were contacted to determine the school attended.

An anonymous questionnaire was distributed to the school on Monday, 6 June 2011. The paper-based questionnaire was delivered to each classroom with an explanatory letter requesting that the questionnaire be completed by parents or caregivers up until Friday, 24 June 2011. It also included CDCB contact details for parents or caregivers with any comments or questions. The questionnaire was also available as an online questionnaire with a link provided in the explanatory letter.

The questionnaire included questions on demographics, details of illness (onset date, symptoms, health care seeking behaviour, management and diagnosis), chronic health conditions and vaccination status. There were 20 questions and it was estimated the questionnaire would take approximately 10 minutes to complete. The questionnaire was open to all students and staff.

Influenza-like illness (ILI) was defined as fever plus cough and/or sore throat as reported in the questionnaire. A descriptive analysis of ILI cases and cases that reported illness not consistent with ILI was undertaken. Attack rates were calculated as the number of students with ILI divided by total student respondents. Due to small numbers, a separate analysis of the 10 staff questionnaires was not undertaken.

Data analysis was performed using Stata 10 software. The relationships between ILI and class and ILI and age were analysed with a chi-squared test. Influenza vaccine effectiveness and the relationship between ILI and chronic lung disease was analysed using a Fisher's exact test.

\section{Microbiological investigation}

Throat or nasal swabs were requested from the initial sample of 10 ill schoolchildren. Swabs were collected either by a collection centre of the state's public health laboratory (SA Pathology) or by the family general practitioner. Swabs were analysed at SA Pathology. Ribonucleic acid (RNA) was extracted from patient samples using the MagMAX ${ }^{\mathrm{TM}}$ automated extraction platform and the MagMax Total nucleic acid extraction kit (Life Technologies, Carlsbad, California, United States of America). Two hundred microlitres $(\mu \mathrm{L})$ of patient sample and $25 \mu \mathrm{L}$ of extraction/inhibition control were used in each extraction and eluted into $100 \mu \mathrm{L}$ of kit buffer. The real-time polymerase chain reaction (PCR) assays used for the amplification and detection of influenza $A$ and $B$ were modified versions of the Centers for Disease Control and Prevention (CDC) real-time reverse transcription PRC (rRTPCR) Protocol for Detection and Characterization of Influenza (version 2007). The inhibition control, a synthetic oligo cloned into the Pgem-t easy vector (Promega, Fitchburg, Wisconsin, United States of America), was amplified in a separate reaction. Additional testing for adenovirus, parainfluenza 1,2 and 3 , respiratory syncytial virus, rhinovirus and human metapneumovirus were performed as single duplex or triplex reactions (Mark Turra unpublished data). The assays were carried out in a single $12.5 \mu \mathrm{L}$ (final volume) influenza $A$ and $B$ multiplex reaction, using the Invitrogen SuperScript III platinum One-Step Quantitative RT-PCR system (Life Technologies, Carlsbad, California, United States of America) and 2.5 $\mu \mathrm{L}$ of eluted RNA. The RNA was amplified using the LC480 real-time cycler (Roche, Basel, Kanton Basel, Switzerland) using the following cycling parameters: $50^{\circ} \mathrm{C}$ for 15 minutes, 95 ${ }^{\circ} \mathrm{C}$ for 10 minutes followed by 45 cycles of $95^{\circ} \mathrm{C}$ for 15 seconds, and $60{ }^{\circ} \mathrm{C}$ for 45 seconds. The second derivative max analysis provided by the LC480 software was used for interpretation of results. Results were interpreted as detected, equivocal or negative (Mark Turra, SA Pathology, personal communication, 5 June 2012).

Five specimens were sent to the Victorian Infectious Diseases Reference Laboratory (a World Health Organization [WHO] Collaborating Centre for Reference and Research on Influenza) for culture to determine subtyping and vaccine match.

Laboratory results were unable to be linked to questionnaire answers as the questionnaires were completed anonymously; therefore, laboratory-confirmed cases were reported separately. Information obtained from the laboratory, doctor notification and/or preliminary interviews on onset dates and symptoms were collated. 


\section{Environmental investigation}

CDCB staff, along with two local council environmental health officers, visited the school on Monday, 30 May 2011.

\section{RESULTS}

\section{Epidemiological investigation}

Questionnaire responses were returned for 100 (55.9\%) of the 179 students at the school. In two instances, two questionnaires were completed on the same questionnaire paper; since it was impossible to distinguish the individual responses, the four students were excluded. Therefore a total of 96 (53.6\%) questionnaires were analysed.

Valid questionnaires were returned for 53 males (mean age 7.5 ) and 43 females (mean age 7.8 ) of which 37 males $(69.8 \%)$ and 35 females $(81.4 \%)$ reported being unwell. A total of 43 students, 25 males (47.2\%) and 18 females (41.9\%), reported ILI, giving an attack rate of illness of $75.0 \%$ and an attack rate of ILI of $44.8 \%$. Students with ILI had a mean age of 7.5 years compared with 7.8 years for those without ILI. There was no association between ILI and age $(P>0.05)$ or ILI and class $(P>0.05)$. There was no apparent relationship between date of ILI onset and age or class (data not shown).

An epidemic curve of the ill cases is shown in Figure 1. The first three ILI cases were all in the same class with subsequent rapid spread to all other classes in the school. All but three cases occurred within a 20-day period (15 May until 3 June 2011). The peak $(n=7)$ occurred on 23 May 2011. Illness duration was longer in students with ILI compared with those without ILI (Figure 2).

Among the 43 students with ILI, constitutional and respiratory symptoms were most common with students reporting the following symptoms: tiredness $(n=38)$, cough $(n=38)$, anorexia $(n=38)$ and sore throat $(n=36)$. Tiredness $(n=17)$, headache $(n=13)$ and rhinorrhea $(n=12)$ were the most common symptoms in students who were unwell but did not satisfy the ILI case definition. Thirty-one students with ILI $(72.1 \%)$ reported abdominal pain, vomiting and/or diarrhoea (Table 1).

A total of 41 students (42.7\%) presented to health care workers as a result of the illness, including 35 cases with ILI ( $81.4 \%$ of all ILI cases). Antibiotics

\section{Figure 1. Epidemic curve by onset date, Adelaide, Australia, 2011}

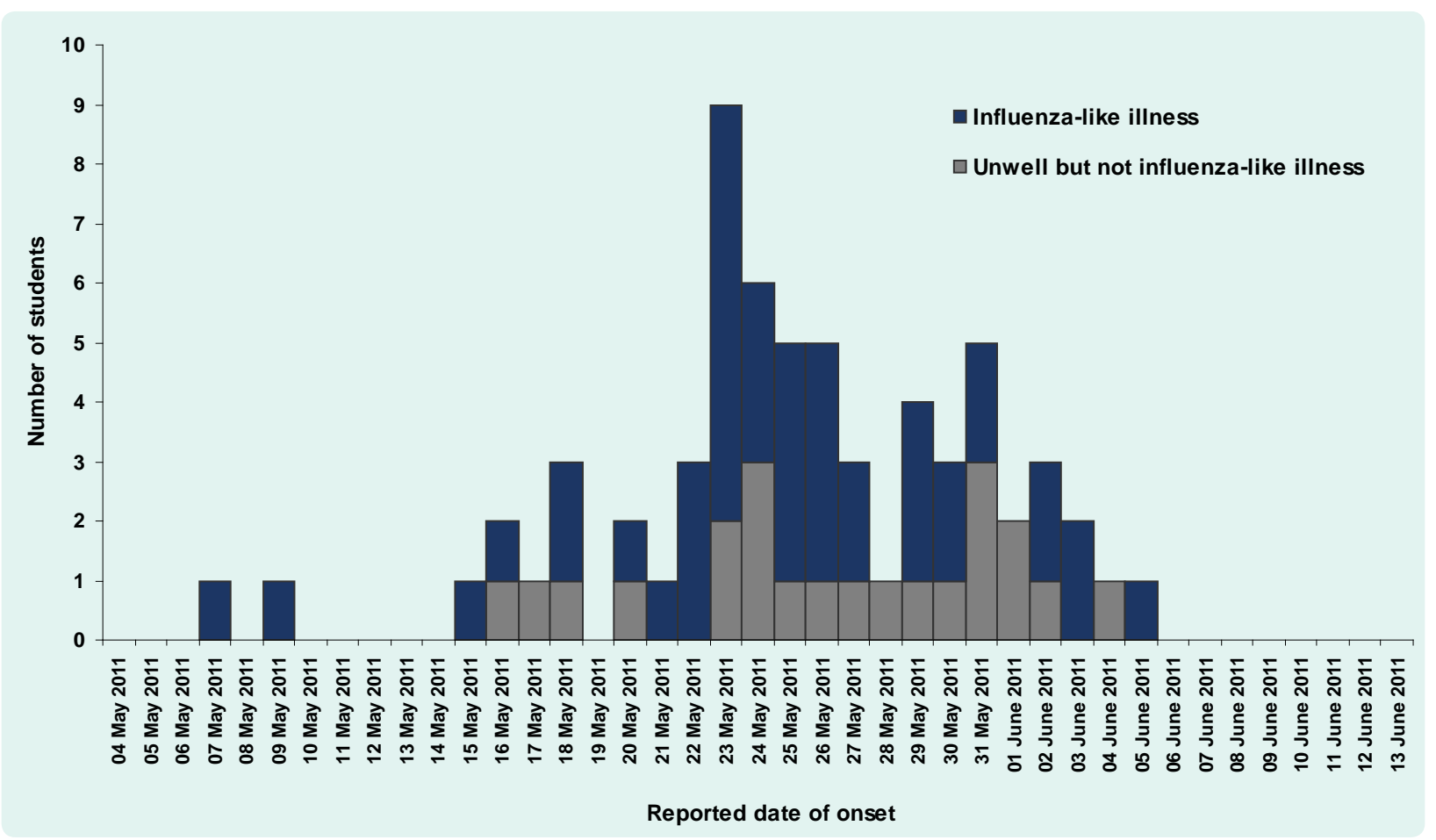


Figure 2. Reported duration of illness in questionnaire respondents who had been unwell, Adelaide, Australia, 2011

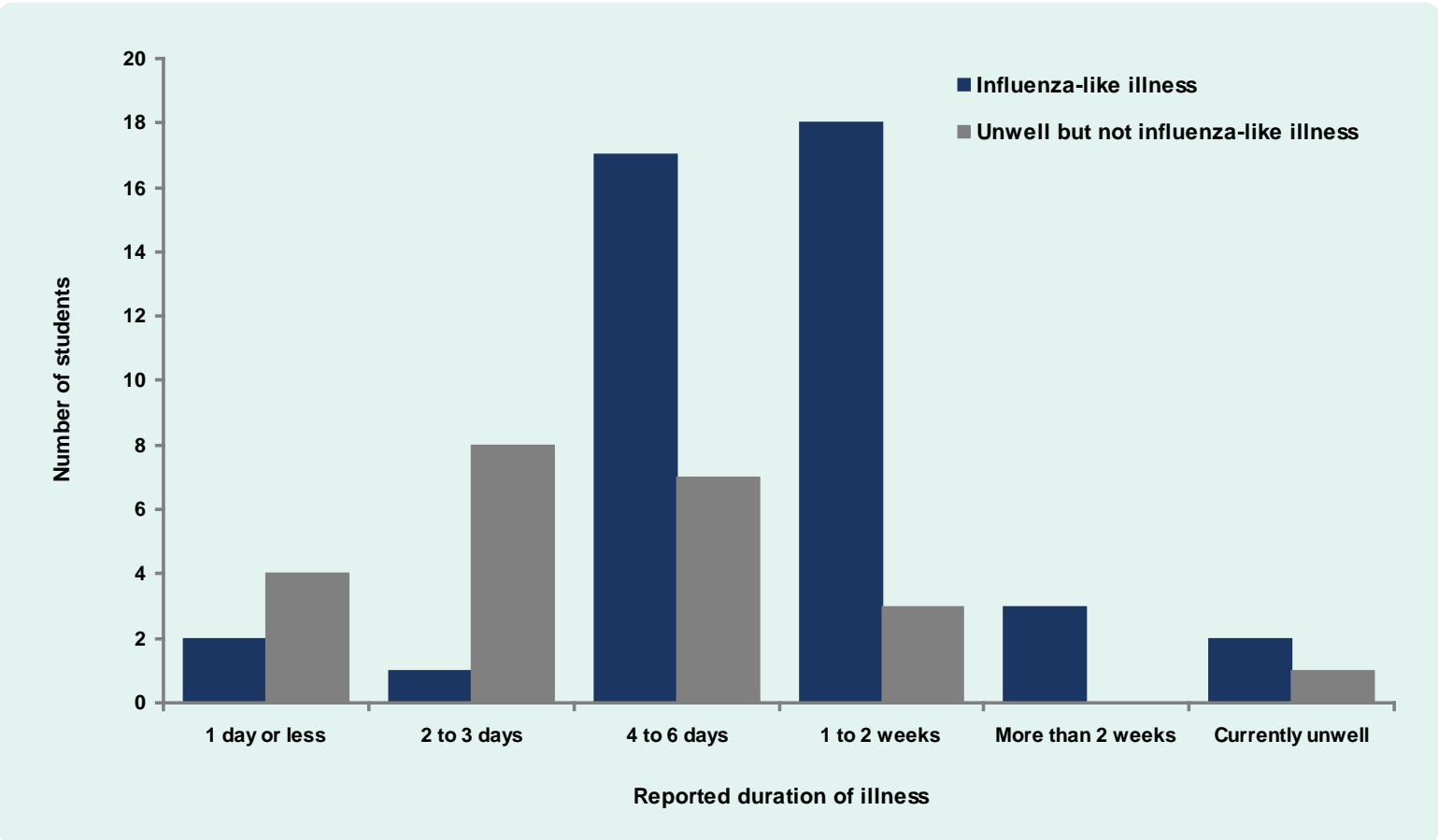

Table 1. Reported symptoms in questionnaire respondents who had been unwell, Adelaide, Australia, 2011

\begin{tabular}{lccc}
\hline Symptoms & $\begin{array}{c}\text { IIIness met ILI } \\
\text { case definition }(\boldsymbol{n}=\mathbf{4 3})\end{array}$ & $\begin{array}{c}\text { Unwell but illness did not meet } \\
\text { ILI case definition }(\boldsymbol{n}=\mathbf{2 9})\end{array}$ & $\begin{array}{c}\text { Number } \\
\text { of respondents }\end{array}$ \\
\hline Fever & 43 & 8 & 67 \\
Tiredness & 38 & 17 & 65 \\
Cough & 38 & 10 & 63 \\
Anorexia & 38 & 8 & 62 \\
Sore throat & 36 & 10 & 62 \\
Headache & 32 & 13 & 62 \\
Rhinorrhea & 32 & 12 & 62 \\
Abdominal pain & 23 & 9 & 62 \\
Nausea & 19 & 3 & 57 \\
Muscle aches & 18 & 0 & 54 \\
Vomiting & 11 & 2 & 53 \\
Diarrhoea & 7 & 1 & 49 \\
Epistaxis & 2 & 1 & 51 \\
\hline
\end{tabular}

were recommended by health care workers for $19 \mathrm{ILI}$ cases.

Lung conditions (e.g. asthma) were the most commonly reported chronic illness among questionnaire respondents $(n=10,10.4 \%)$. There was no association between ILI and chronic lung illness (relative risk [RR]: 0.93, 95\% confidence interval [Cl]: 0.44-1.95, $P=1.00)$.
Ninety-four students responded to the question regarding vaccination with six $(6.4 \%)$ reporting vaccination in 2011 before illness onset in the school and an additional two reporting vaccination as a result of the outbreak. Four of these reported illness in this outbreak, although none satisfied the ILI case definition. The risk difference for ILI in respondents vaccinated in 2011 before the outbreak was $-0.66(95 \% \mathrm{Cl}:-0.78$ to $-0.54, P=0.05, \mathrm{RR}$ : indeterminate as no cases 
Table 2. Date of onset, symptoms and swap type in students with confirmed influenza B, Adelaide, Australia, 2011

\begin{tabular}{|c|c|c|c|}
\hline Onset date & Reported symptoms & Swab type & Swab collection date \\
\hline 25 May 2011 & Fever, headache, cough, anorexia, diarrhoea & Throat & 1 June 2011 \\
\hline 26 May 2011 & Fever, nausea, sore throat, lethargy, headache, cough & Throat & 31 May 2011 \\
\hline 27 May 2011 & $\begin{array}{l}\text { Fever, sore throat, cough, rash, aches, abdominal pain, lethargy, } \\
\text { rhinorrhea }\end{array}$ & Nasal & 31 May 2011 \\
\hline 28 May 2011 & Fever, sore throat, headache, cough, aches & Throat & 2 June 2011 \\
\hline 30 May 2011 & Cough, influenza-like illness & Nasal & 2 June 2011 \\
\hline 31 May 2011 & Influenza-like illness & Unknown & 31 May 2011 \\
\hline 1 June 2011 & Fever, cough, cold-like symptoms & Throat & 3 June 2011 \\
\hline
\end{tabular}

in immunized), although given the small numbers this should be interpreted with caution.

\section{Microbiological investigation}

On 1 June 2011, the first throat swab was confirmed as influenza B. By outbreak conclusion on 15 June 2011, seven students were swab-positive for influenza B, one student for rhinovirus, and one student for metapneumovirus; one student was swab-negative. Two of the influenza specimens were confirmed as B/Brisbane/60/2008-like (included in the 2011 vaccine) and three specimens were unable to be cultured. Cases with confirmed influenza B satisfied the ILI case definition (Table 2).

\section{Environmental investigation}

Discussion with school staff identified that cough, lethargy and fever were predominant symptoms. Infection control practices including cough etiquette, hand washing, cleaning practices and staying home if unwell were discussed. 'Wash, wipe, cover' posters that promoted infection control were sent to the school. Events involving mixing with other schools were postponed.

The school had seven classes: reception (first year of school); two combined reception/year one classes; a year two class; a combined year two/three class; a combined year three/four class; and a combined year four/five class. There were between 17 and 30 students per class. The two classes where the outbreak appeared to initiate shared a double classroom with removable partition. There was one toilet block. There was a single playground for all students with mixing among year levels.
Daily updates on absentee numbers were obtained from the school, with 40,44, 43, 28, 31, 26 and 23 students absent on 26 May, 27 May, 30 May, 31 May, 1 June, 2 June and 3 June, respectively. By 9 June 2011, absentee numbers had returned to background (two absent).

\section{DISCUSSION}

This outbreak investigation of influenza $B$ in a primary school demonstrated a high attack rate of illness and of ILI. There was no association between ILI and age or chronic lung disease, a low self-reported influenza vaccination rate $(6.4 \%)$ and no reported ILI in respondents vaccinated for influenza prior to the outbreak.

The attack rate of ILI in this study (44.8\%) was lower than the age-specific ILI attack rate of 70\%-80\% reported in a primary school seasonal influenza $A$ outbreak, ${ }^{5}$ but higher than the $34 \%$ attack rate reported in another primary school influenza outbreak ${ }^{6}$ and the $13 \%$ attack rate reported in a secondary school. ${ }^{7}$ Variability in attack rate in reported studies may reflect seasonal differences in influenza virus behaviour, environmental conditions and population exposure history.

The attack rate of any illness in student respondents in this study was $75.0 \%$. The cause of illness in unwell students who did not satisfy the ILI case definition is unknown; however, as influenza can be asymptomatic or mildly symptomatic, it is possible that these students had influenza. ${ }^{2}$ High absentee numbers occurred for more than two weeks with a peak on 27 May 2011 with 44 students absent $(24.6 \%$ of total students).

The majority of students with ILI had gastrointestinal symptoms in addition to respiratory symptoms. 
This has also been reported in a school outbreak of influenza A (e.g. nausea in $61 \%$ and vomiting in $45 \%$ of students). ${ }^{6}$ Gastrointestinal disorders have also been reported as being significantly more common in children with influenza B compared with influenza A $(64 \%$ versus $39 \%, P=0.03) .^{2}$ The atypical presentation of influenza in children may contribute to the spread of influenza in the community through reduced recognition of influenza as the cause of illness as occurred in this outbreak. ${ }^{2}$

In this study, $10.4 \%$ of children were reported to have chronic lung illness (including asthma), which is similar to the percentage given in the National Health Survey 2004-2005. ${ }^{12}$ There was no association between ILI and chronic lung illness in this study. This may be due to the small number of cases and consequent variability of estimates; an alternative explanation is that there was no association between development of ILI and pre-existing chronic lung disease, only with development of severe ILI. ${ }^{13}$

The inactivated trivalent influenza vaccine is used in Australia. The vaccine used at the time of the outbreak was against the following strains: A/California/7/2009 (H1N1)-like virus, A/Perth/16/2009 (H3N2)-like virus and B/Brisbane/60/2008-like virus. ${ }^{14}$ Hence, the vaccine matched the strain seen in this outbreak. None of the six questionnaire respondents vaccinated in 2011 against influenza before this outbreak developed ILI. This corresponds to a vaccine effectiveness, from questionnaire data, of $100 \%$ against ILI. However, the use of proxy report without validation, the small size of the school and a response rate of $53.6 \%$ may have resulted in a falsely elevated estimation of vaccine effectiveness and so this result should be interpreted with caution. It is possible that use of ILI as a proxy for influenza underestimated influenza through vaccineassociated alteration of the influenza symptom profile. ${ }^{15}$ This may result in a differential presentation of influenza with an increased number of vaccinated students with asymptomatic or atypical influenza infection which did not meet the ILI definition, compared with a more typical presentation in unvaccinated students. ${ }^{15}$

In Australia, influenza vaccine is recommended for anyone from six months of age who wishes to be protected against influenza; however, it is provided free for children with specified chronic illnesses. ${ }^{16}$
To the authors' knowledge there is no formal estimate of influenza vaccine coverage in children in Australia, although non-peer-reviewed literature estimated influenza vaccine coverage in children at $10 \% .{ }^{17}$ Our study found a self-reported influenza vaccination rate for students of $6.3 \%$ before the outbreak and $9.6 \%$ after the outbreak. This so provides an estimate for vaccine coverage for school-aged children in 2011. Despite the recent $\mathrm{H} 1 \mathrm{~N} 1$ influenza pandemic, our study found low estimated influenza vaccine coverage in this age group. Influenza vaccine coverage may have been influenced by media reports of side-effects associated with Fluvax and Fluvax Junior vaccine in $2010 .^{17,18}$ Further studies to estimate influenza vaccination coverage in Australian children are required.

This study has several limitations. Questionnaire results were obtained by proxy from parents or caregivers with no validation of responses, which may have resulted in measurement error. There was possibility of selection bias as caregivers of ill students may have been more likely to respond, and there was considerable variation in response between classes. The small size of the school and response rate resulted in small numbers on which to base conclusions regarding vaccination coverage and effectiveness. Several questionnaires were incomplete, particularly regarding symptoms with several respondents only answering questions in the affirmative and leaving other subparts blank. Some parents or caregivers may have been from a culturally and linguistically diverse background and been unable to complete the questionnaire or complete it accurately; however, given the anonymous nature of the questionnaire and the use of the school to distribute the questionnaire, it is impossible to determine the effect. It is possible, although unlikely, that respondents completed more than one questionnaire, as questionnaires were not identification coded. The study strengths included documentation of an outbreak of seasonal influenza in a school including information on symptoms and vaccination history and a reasonable response rate to the cohort questionnaire.

This cohort study has characterized an influenza B outbreak in a school. As such, it has estimated the attack rate, influenza vaccine coverage and influenza effectiveness within the setting of a primary school-aged cohort in addition to characterizing ILI in children, which commonly included gastrointestinal symptoms. 


\section{Conflicts of interest}

None declared.

\section{Funding}

None.

\section{Acknowledgements}

We would like to thank all those involved with the investigation and control of this outbreak including Dr Ann Koehler; staff of the Communicable Disease Control Branch; and the staff and students of the school.

\section{References:}

1. Bridges CB et al. Influenza. In: Heymann DL, editor. Control of communicable diseases manual. $19^{\text {th }} \mathrm{ed}$. Washington DC, American Public Health Association, 2008, 315-331.

2. Aymard M et al, Sentinel Physicians from the Grippe et Infections Respiratoires Aiguës Pédiatriques Network. Burden of influenza in children: preliminary data from a pilot survey network on community diseases. Pediatric Infectious Disease Journal, 2003, 22 Suppl:S211-214. pmid:14551477

3. Reichert TA et al. The Japanese experience with vaccinating schoolchildren against influenza. The New England Journal of Medicine, 2001, 344:889-896. doi:10.1056/ NEJM200103223441204 pmid:11259722

4. Heikkinen T et al. Burden of influenza in children in the community The Journal of Infectious Diseases, 2004, 190:1369-1373. doi: 10.1086/424527 pmid:15378427

5. Selden S, Cameron S. Influenza A outbreak at a Mount Gambier junior primary school, South Australia. Communicable Diseases Intelligence, 1994, 18:357-360.

6. Brock C, Knowles M, Goh S. A school and community outbreak of influenza A. Communicable Disease Report, CDR Review, 1995, 5(12):R177-179. pmid:8541938

7. Danis $\mathrm{K}$ et al. Lessons from a pre-season influenza outbreak in a day school. Communicable Disease and Public Health, 2004, 7:179-183. pmid:15481209
8. South Australian Public and Environmental Health Act 1987. Adelaide, Government of South Australia, 2012 (http:// www.legislation.sa.gov.au/LZ/C/A/PUBLIC\%20AND\%20 ENVIRONMENTAL\%2 OHEALTH\%20 ACT\%201987/ CURRENT/1987.36.UN.PDF, accessed 24 May 2012).

9. South Australian Public and Environmental Health (Notifiable Diseases) Regulations 2004. Adelaide, Government of South Australia, 2009 (http://www.legislation.sa.gov.au/LZ/C/R/ PUBLIC\%20AND\%20ENVIRONMENTAL\%20HEALTH\%20 (NOTIFIABLE\%20DISEASES)\%20REGULATIONS\%202004/ CURRENT/2004.181.UN.PDF, accessed 24 May 2012).

10. Flood L. Disease surveillance and investigation report 1 July to 31 December 2010. Public Health Bulletin SA, 2011, 8(1):62-7311.

11. Communicable Disease Control Branch. Disease notification: 7 year and YTD comparisons 2012. Adelaide, Government of South Australia, 2012 (http://www.dh.sa.gov.au/pehs/notifiablediseases-summary/weekly\%20report\%203\%20120602.pdf, accessed 18 June 2012).

12. Australian Centre for Asthma Monitoring. Asthma in Australia 2008. Canberra, Australian Institute for Health and Welfare; 2008 (http://www.asthmamonitoring.org/AinA08_html/Index. htm, accessed 30 March 2012).

13. Hirota $Y$ et al. Various factors associated with the manifestation of influenza-like illness. International Journal of Epidemiology, 1992, 21:574-582. doi:10.1093/ije/21.3.574 pmid:1634321

14. Recommended composition of influenza virus vaccines for use in the 2011 southern hemisphere influenza season. Geneva, World Health Organization (http://www.who.int/influenza/ vaccines/virus/recommendations/recommendations2011south/ en/\#, accessed 30 March 2012).

15. Ehrlich $\mathrm{HJ}$ et al. A cell culture-derived influenza vaccine provides consistent protection against infection and reduces the duration and severity of disease in infected individuals. Clinical Infectious Diseases, 2012, 54:946-954. doi:10.1093/cid/ cir959 pmid:22267715

16. Questions and answers for influenza (flu) vaccination. Canberra, Australian Government Department of Health and Ageing, 2012 (http://www.immunise.health.gov.au/internet/immunise/ Publishing.nsf/content/immunise-influenza-qanda, accessed 30 March 2012).

17. Fitzgerald D. Influenza vaccination in kids. Medical Observer, 2011 (http://www.medicalobserver.com.au/news/influenza-vaccinationin-kids, accessed 6 September 2011).

18. Blyth CC et al. Ensuring safety of the 2011 trivalent influenza vaccine in young children. Medical Journal of Australia, 2011, 195:52. pmid:21728948 